\title{
Application of neural networks to laser ultrasonic NDE of bonded structures
}

\author{
C.-D. Tsai, T.-T. Wu*, Y.-H. Liu \\ Institute of Applied Mechanics, College of Engineering, National Taiwan University, Taipei 106, Taiwan
}

Received 15 July 2000; revised 20 December 2000; accepted 1 February 2001

\begin{abstract}
The purpose of this study is to develop artificial neural networks for the nondestructive evaluation of a bounded structure using laser generated surface waves. The radial basis model is employed and the inputs of the networks are the sampled dispersion curves while the output is the thickness of the bonding layer or unbond ratio. Numerical simulated dispersion curves with noises were used to train and test the neural networks. The dispersion curves of laser ultrasonic experiments acquired by spectral analysis method were also utilized to test the systems. For comparison, simplex inverse method was also introduced. The results demonstrate that neural networks are well suited for online evaluation of bonding thickness and unbond ratio of a bonded layered structure. (C) 2001 Published by Elsevier Science Ltd.
\end{abstract}

Keywords: Neural network; Dispersion curve; Laser ultrasonics ; Bonding quality

\section{Introduction}

Due to the broad use of composite structures, the nondestructive evaluation (NDE) of bonding structures has gathered a lot of interest, in particular, the detection of delamination and/or bonding quality in bonded structures is of practical importance. Due to the noncontact feature and the ability of broadband signal generation, laser ultrasonics has been utilized to investigate the wave propagation phenomena in composite structures [1-6]. Further, it has also been applied to the NDE of elastic properties and/or defects [7-10] in various structures, and promising results were obtained.

On the other hand, it is known that neural networks possess benefits of real-time and noise tolerance, and therefore, they are most suitable for in situ nondestructive evaluation of materials. Lorenz and Wielinga [11] utilized perceptron networks to detect the shape of defects in steel. The inputs were ultrasonic signals and the outputs indicated the shape of the defect. Thus more information was provided in the event that the B-scan image resolution was not enough. Kitahara et al. [12,13] applied a threelayer back propagation network to detect depth of cracks in steel plate. The inputs were ultrasonic time signal and frequency spectrum. The outputs were 10 neurons, which

\footnotetext{
* Corresponding author. Tel.: +886-2-2363-0979; fax: +886-2-23639290.

E-mail address: wutt@spring.iam.ntu.edu.tw (T.-T. Wu).
}

represented 10 kinds of crack depths. Williams and Gucunski [14] introduced the use of neural networks to evaluate pavement thickness and properties, which was trained with results of many numerical simulation of waves propagation with different layers configuration.

Recently, Wu and Liu [15] demonstrated that the thickness and elastic properties of bonding layer in a bonded structure could be determined by utilizing the inverse optimization method. As to the detection of the unbond region, $\mathrm{Wu}$ and Chen [16] utilized the wavelet transform to study the laser generated surface waves in a bonded structure. They found that the dispersion analysis of surface waves can be employed to differentiate the unbonded from the bonded regions in layered structures.

The purpose of this paper is an extension of the previous work $[15,16]$ to develop an efficient method for rapid nondestructive evaluation of bonded structures based on neural networks. Radial basis neural networks are employed in this paper to evaluate the thickness of a bonding layer and/or unbond area of a bonded structure. The inputs are sampled dispersion curves while the output presents the bonding thickness or unbond ratio. Training and testing sets are composed of many numerical simulations of surface wave dispersion in layered media. To evaluate the system performance, laser ultrasonic experiments were performed on an epoxy bonded copper-aluminum layered specimen with very small bonding thickness. The measured dispersive elastic wave signals were processed via the spectral analysis method to obtain the experimental dispersion curve. 


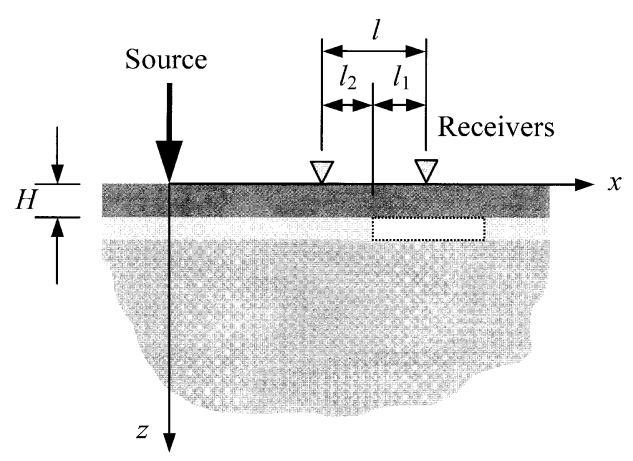

Fig. 1. Surface analysis of surface wave test of layered medium.

\section{Surface wave dispersion in bonded layered structures}

\subsection{Calculation of surface wave dispersions}

The propagation of surface wave in an isotropic layered half space has been studied by many researchers and the associated references can be found in the book by Aki and Richard [17]. The following calculations of the dispersive surface waves in layered media were calculated by a general-purpose computer program, which was written based on the matrix method $[18,19]$ for the calculations of dispersion curves of isotropic as well as anisotropic multilayered media.

Shown in Fig. 1 is an epoxy bonded layered medium with part of the area unbonded. The thickness of the surface layer is $H$. In the figure, transient elastic waves are generated by a point source (in this paper, a Nd-YAG pulsed laser was used) and detected by a pair of receivers with spacing $l . l_{1}$ and $l_{2}$ are the unbond and the bond parts of $l$, respectively. For the elastic wave propagating through both the bond and unbond regions, the velocity dispersion cannot be predicted from either the Lamb wave analysis or the generalized surface wave analysis. However, to the first order approximation, it can be assumed that the elastic wave propagation in the unbond region is with the velocity of Lamb wave and that in the well bond region is with the velocity of the generalized surface wave [16], i.e.

$$
v(\omega)=\frac{l_{1}+l_{2}}{\frac{l_{1}}{v_{1}(\omega)}+\frac{l_{2}}{v_{2}(\omega)}}
$$

where $l_{1}$ and $l_{2}$ are the propagation distances in the unbond region and well bond region, respectively, $v_{1}$ and $v_{2}$ are the velocities of the fundamental anti-symmetric Lamb mode and the generalized surface wave mode, respectively.

It is worth noting that as the laser point source or the receiver is very close to the boundary of the well bonded and unbonded regions, the diffraction induced by the discontinuity is not considered in Eq. (1).

\subsection{Dispersion analysis of surface waves}

Consider the test configuration shown in Fig. 1. Transient elastic waves are generated by a point source and detected by a pair of receivers. The cross-power spectrum, $\bar{S}_{x_{1}, x_{2}}(f)$, between the two signals is defined as

$\bar{S}_{x_{1}, x_{2}}(f)=\frac{1}{n} \sum_{i=1}^{n}\left\{\left[R_{1}(f)\right]_{i} \cdot\left[R_{2}^{*}(f)\right]_{i}\right.$

where $R_{1}(f)$ and $R_{2}(f)$ correspond to the Fourier transforms of time records from the two receivers located a distance $l$ apart, while $n$ is the number of records averaged. The phase difference $\phi$ of the two signals can be calculated from

$\phi=\tan ^{-1} \frac{\operatorname{Im}\left[\bar{S}_{x_{1}, x_{2}}(f)\right]}{\operatorname{Re}\left[\bar{S}_{x_{1}, x_{2}}(f)\right]}$

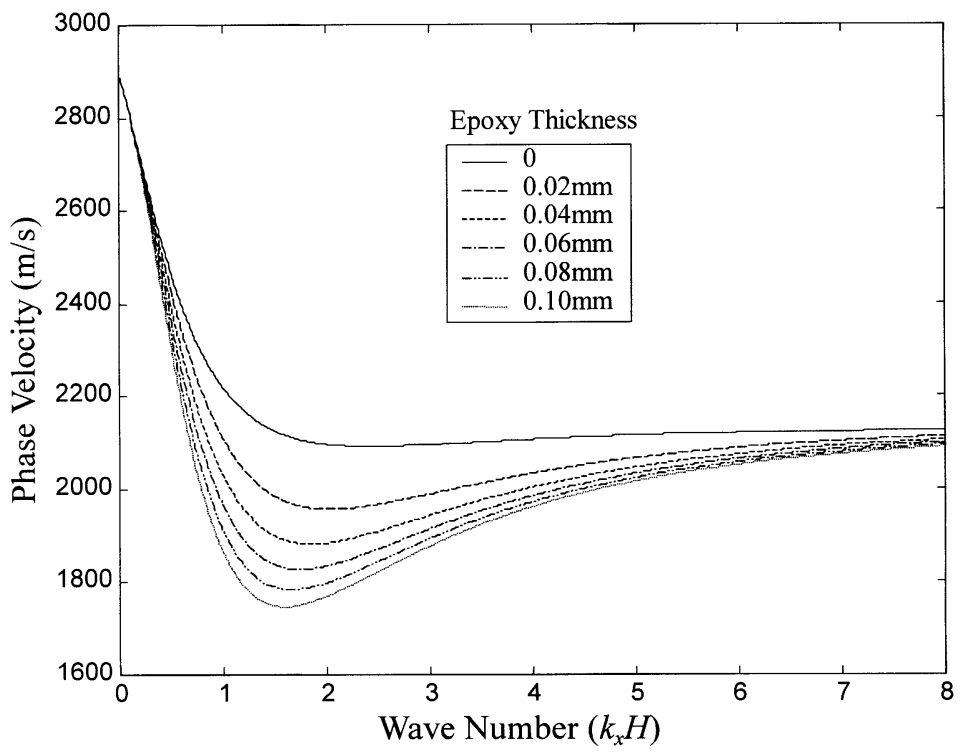

Fig. 2. Numerical dispersions of various epoxy thicknesses. 


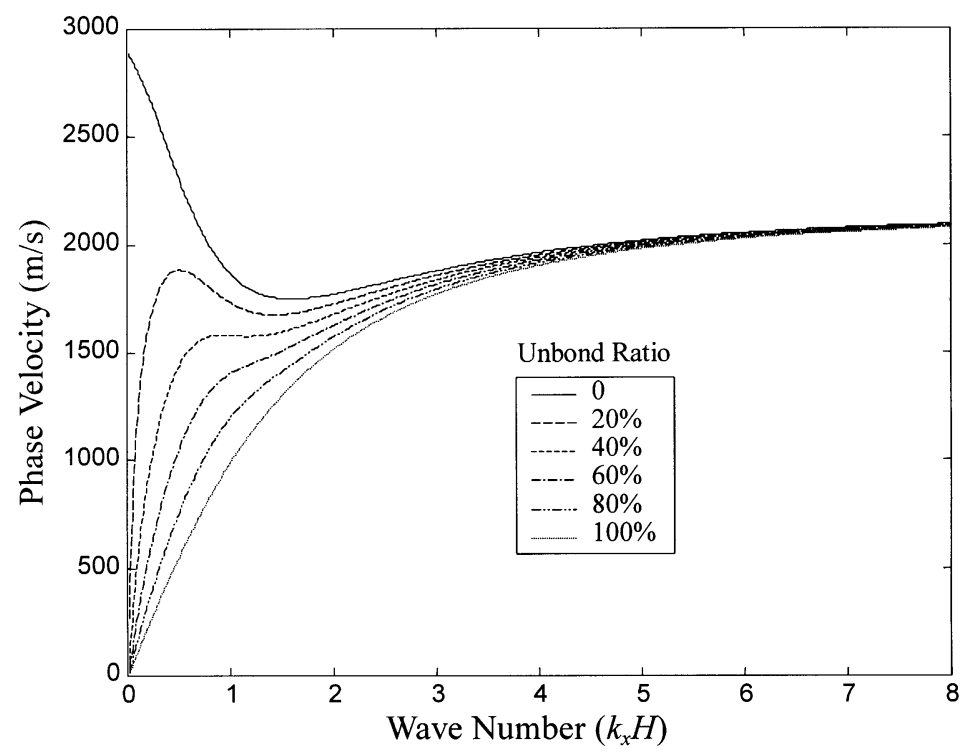

Fig. 3. Numerical dispersions of various unbond ratios.

and the phase velocity $V_{\mathrm{ph}}$ is thus obtained by

$V_{\mathrm{ph}}=2 \pi f \frac{l}{\phi}$

\section{Neural networks}

Neural networks are composed of many simple elements operating in parallel. These elements are inspired by biological nervous systems. The network function is determined largely by the connections between the elements [20]. The weighted data accepted from input layer or previous layer are summed by neuron elements and then are transformed and propagated to output layer or next layer. Through learning rules of various neural models, the weightings are properly adjusted. The neural network can then respond to the input data accurately and rapidly.

\subsection{Abstraction of features}

In order to provide useful features as the input vector of neural network, we must consider the following rules [21]:

1. The elements of the feature vector must be the typical features so that the feature vector differs from each other evidently.

2. Only the best features are abstracted to decrease the dimension of the feature vector and the complexity of the system.

For an epoxy-bonded copper-aluminum layered specimen, the dispersion curves of various bonding thickness and unbond ratio can be calculated using the general purpose program described in Section 2 and are shown in Figs. 2 and 3 , respectively, where $k_{x}$ is the wave number of surface wave and $H$ is the thickness of the surface layer. Figs. 2 and 3 show that the shape of the dispersion curve is very sensitive to the thickness of the bonding layer and the unbond ratio, therefore, the sampled dispersion curve is suitable for the feature vector. We note that for $100 \%$ (the lowest curve in Fig. 3) unbond ratio, this is corresponding to the Lamb wave propagation in a thin plate. For the unbond ratio equal to zero (the highest curve in Fig. 3), the dispersion curve is equivalent to that of a perfect bonded layered half-space. For higher frequency or larger wave number, such as $k_{x} H>4$ in Fig. 3, the phase velocities converge to the Rayleigh wave velocity of surface layer. Therefore, the influence of the bonding layer on the dispersion of surface wave becomes small. On the other hand, we note that the experimental dispersion curve is usually noisier in the low frequency or smaller wave number region, such as $k_{x} H<0.5$. Consequently, the phase velocity dispersions of $k_{x} H$ between 0.5 and 4 sampled in 0.1 were picked as 36 features in this study.

\subsection{Neuron model and network architectures}

In general, the multi-layered back-propagation network (BPN) is the most commonly-used model. However, the determination of layer number and neuron number of each hidden layer is quite difficult. In order to choose the best parameters, lots of training is required. On the other hand, the radial basis neural networks (RBN) require less training time. The neuron number of hidden layer is automatically determined during training procedure. Therefore the RBN is chosen to be the network model in this paper.

Fig. 4 shows the RBN model which is made of only two layers of neurons, namely hidden layer and output layer. The transfer function of hidden layer is a Gaussian function while that of the output layer is a linear function. The net 


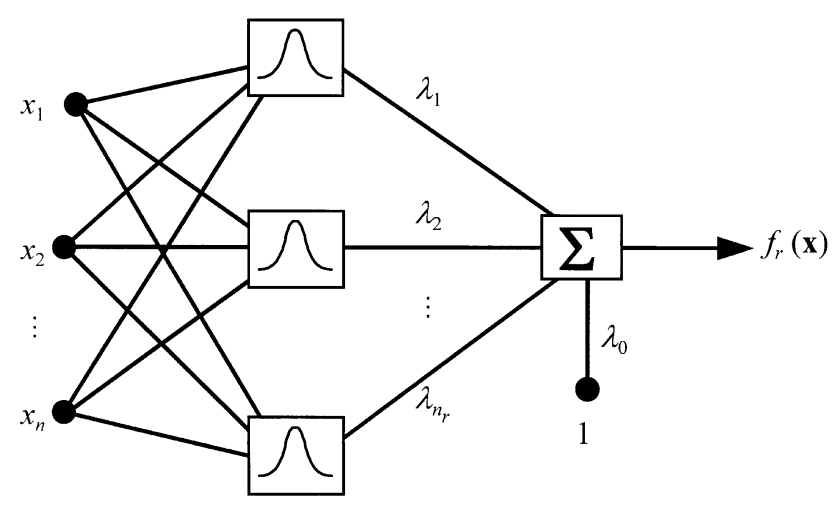

Fig. 4. Architecture of radial basis neural (RBN) net model.

input to the hidden layer is the vector distance between its weight vector $\mathbf{c}_{i}$ and the input vector $\mathbf{x}$, multiplied by the bias $\lambda_{i}$. The output function $f_{r}(\mathbf{x})$ of the $r$ th output neuron may be described as

$f_{r}(\mathbf{x})=\lambda_{0}+\sum_{i=1}^{n_{r}} \lambda_{i} \phi\left(\left\|\mathbf{x}-\mathbf{c}_{i}\right\|\right)$

where $n_{r}$ is the neuron number of hidden layer; $\phi(0)$ is the Gaussian function. Through an orthogonal least squares learning algorithm [22] the weighting and bias are directly evaluated with least sum-square output error. The neuron number of hidden layer may be increased as well as training epoch increases until output error decreases to an acceptable value. The neuron number of hidden layer is then determined.

In this paper, the input vector is composed of 36 abstracted features adopted from the dispersion curve while the output is a single neuron, which indicates the bonding thickness or unbond ratio. As the bonding thickness must be larger than zero and the unbond ratio must be between zero and $100 \%$, the network output will be rearranged if it exceeds these limits.

\subsection{Training procedures}

To evaluate bonding thickness, the dispersion curves with $0,0.01 H, 0.02 H, \ldots, 0.1 H$ of bonding layer thickness were utilized. To improve the noise tolerance, uniformly distributed random numbers between $(-0.1 \pi$ and $0.1 \pi)$ were added to phase angle of 10 copies of the above 11 signals. Therefore, in all there are 110 signals utilized in the training set. Another 11 signals with $0,0.005 H, 0.015 H$, $0.025 H, \ldots, 0.105 H$ bonding layer thickness were generated as the testing set. Similar consideration of the noise treatment as that in the training set was taking in the testing set. During the training phase, the network error converges, as shown in Fig. 5. The solid and dashed lines demonstrate the error of the training set and testing set, respectively. The result shows that the least testing error can be achieved with a spread of 1586 in the Gaussian function and 14 epochs. That is, 15 hidden layer neurons were required in this neural network.

To evaluate the unbond ratio, the dispersion curves with $0,10,20, \ldots, 100 \%$ of unbond ratio and $5,15,25, \ldots, 95 \%$ of unbond ratio were utilized to generate 110 training sets and 100 different testing sets, respectively, according to the above procedures. The network error during training converges is shown in Fig. 6. The result shows that the least testing error can be achieved with a spread of 6000 in the Gaussian function and 5 epochs. That is, six hidden layer neurons were required in this neural network.

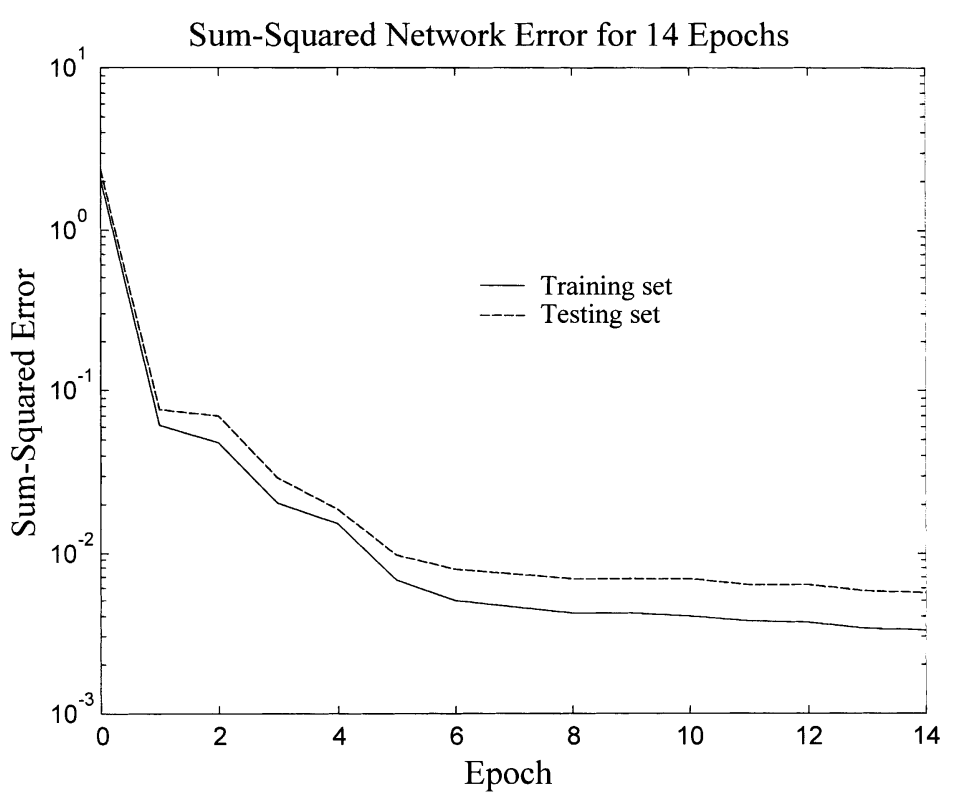

Fig. 5. Network error for evaluating of layer thickness. 


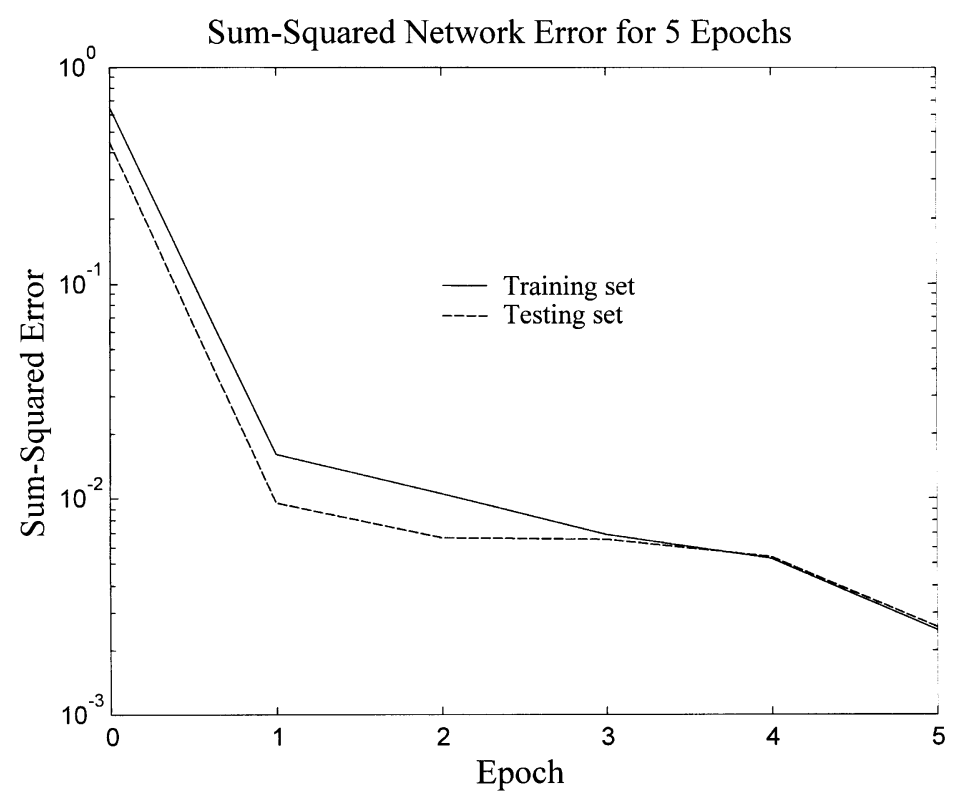

Fig. 6. Network error for evaluating of unbond ratio.

\section{Measurements of dispersion curves using laser generated surface waves}

\subsection{Experimental set-up}

The experimental configuration of the present study is shown in Fig. 7, where the focused ND:YAG pulsed laser beam (Quanta-Ray, GCR-130) (wavelength $532 \mathrm{~nm}$ ) provides a broadband source. The duration of the laser pulse utilized was $10 \mathrm{~ns}$ and the energy carried was about $100 \mathrm{~mJ}$. The layered specimen was rested on a precision translation stage to accurately control the distance between the source and the receiver. An NBS conical transducer was utilized to measure the generated elastic wave signals from the laser sources. The received voltage signals from the

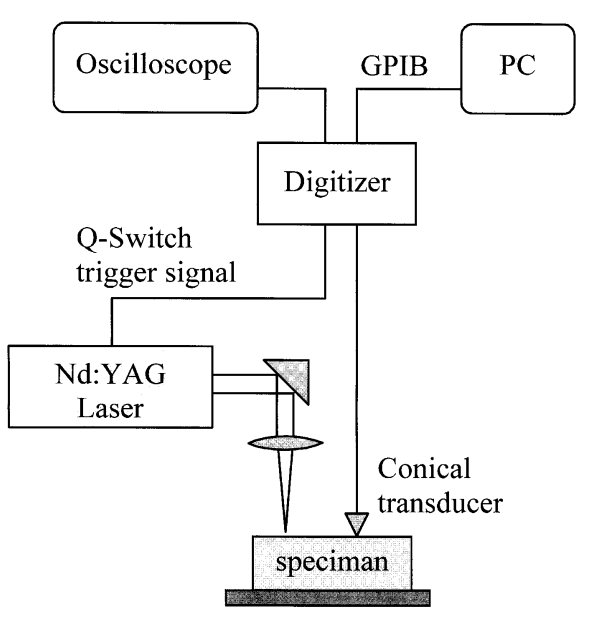

Fig. 7. Experimental configuration of the laser ultrasonic experiment. conical transducer were then amplified by a preamplifier and recorded by a $100 \mathrm{MHz}$ digital oscilloscope (LeCroy 9314L). A trigger signal synchronized with the laser source was utilized to trigger the digital oscilloscope. The recorded signals were sent to a personal computer via GPIB for further spectral analysis.

The layered medium specimen was composed of a $200 \times 200 \times 1 \mathrm{~mm}^{3}$ copper plate and a $200 \times 200 \times 150 \mathrm{~mm}^{3}$ aluminum block. A $1 \mathrm{~mm}$ deep shallow square slot (area $120 \times 80 \mathrm{~mm}^{2}$ ) was machined from the aluminum block. The thin copper plate with $1 \mathrm{~mm}$ thickness was then bonded carefully on top of the aluminum block with epoxy resin. The wave velocities of these materials were measured by the ultrasonic experiments and the results are shown as follows:

- Copper:

$$
\begin{aligned}
& \rho=8500 \mathrm{~kg} / \mathrm{m}^{3} \\
& C_{p}=4848.4 \mathrm{~m} / \mathrm{s} \\
& C_{s}=2271.4 \mathrm{~m} / \mathrm{s}
\end{aligned}
$$

- Aluminum:

$$
\begin{aligned}
& \rho=2698 \mathrm{~kg} / \mathrm{m}^{3} \\
& C_{p}=6389.1 \mathrm{~m} / \mathrm{s} \\
& C_{s}=3097.8 \mathrm{~m} / \mathrm{s}
\end{aligned}
$$

\subsection{Measurements of dispersion curves}

Since the pulsed laser utilized in this study generates stable point sources, instead of using one point source and two receivers, one point receiver and two point sources were utilized. The advantage of this alternative is that only one receiver is needed in the experiment, further, the precision of the change of the source to receiver distance can be 


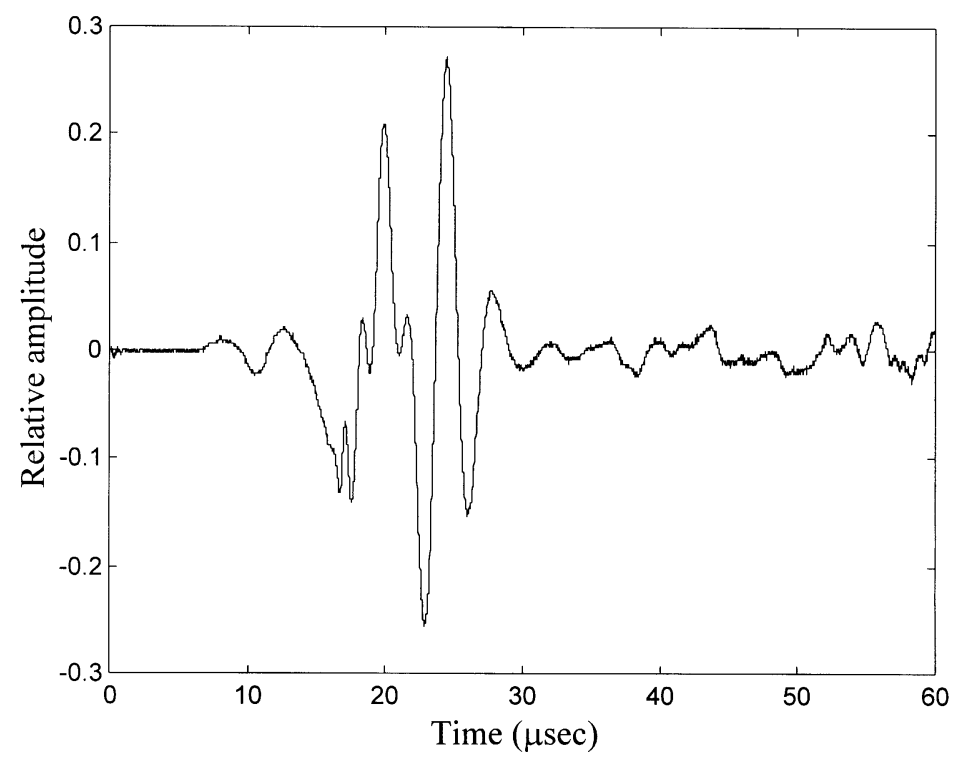

Fig. 8. Wave signal received on the surface of well-bonded zone (source-receiver distance is $35 \mathrm{~mm}$ ).

controlled by a precise translation stage. However, it is worth noting that with this arrangement, the received surface responses of the two receivers located at a distance $x$ are generating from different laser point sources. Therefore, the cross-power spectrum defined in Eq. (2) cannot be applied directly. In this study, the cross-power spectrum is redefined as:

$\bar{S}_{r 1, r 2}(f)=\frac{1}{n_{1}} \sum_{i=1}^{n_{1}}\left[\hat{R}_{1}(f)\right]_{i} \cdot \frac{1}{n_{2}} \sum_{i=1}^{n_{2}}\left[\hat{R}_{2}^{*}(f)\right]_{i}$

where $n_{1}$ and $n_{2}$ are the number of the two sets of records averaged. $\hat{R}_{1}(f)$ and $\hat{R}_{2}(f)$ correspond to the normalized $R_{1}(f)$ and $R_{2}(f)$, respectively. The phase velocity $V_{\text {ph }}$ may be obtained through Eqs. (3) and (4) in the same way as those described in Section 2.

Shown in Fig. 8 is a typical wave signal (displacement perpendicular to the sample surface) received in the wellbonded region of the layered specimen. The distance between the source and the receiver is $35 \mathrm{~mm}$. The vertical axis of Fig. 8 represents the relative amplitude of the displacement signal. Fig. 9 shows a similar wave signal with source to receiver distance equal to $55 \mathrm{~mm}$. We note that due to the dispersion of waves in the layered specimen, big differences in the received signals at difference locations can be found easily from Figs. 8 and 9 .

In the following experiments, the source-receiver offsets for the two receivers shown in Fig. 1 are $x_{1}=35 \mathrm{~mm}$ and

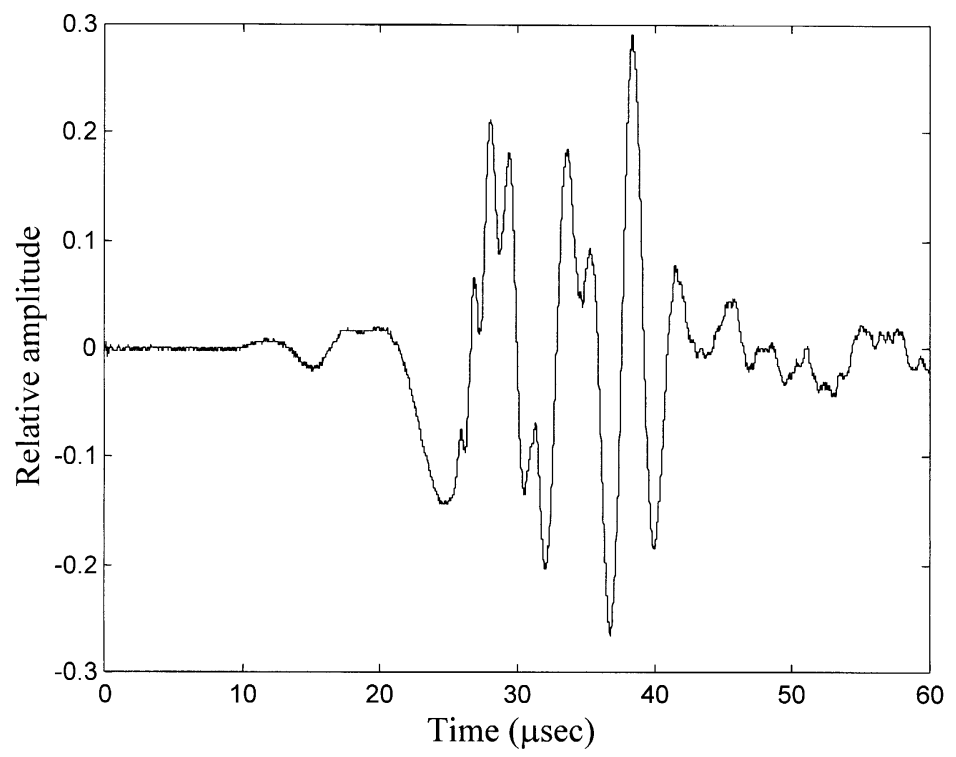

Fig. 9. Wave signal received on the surface of well-bonded zone (source-receiver distance is $55 \mathrm{~mm}$ ) 


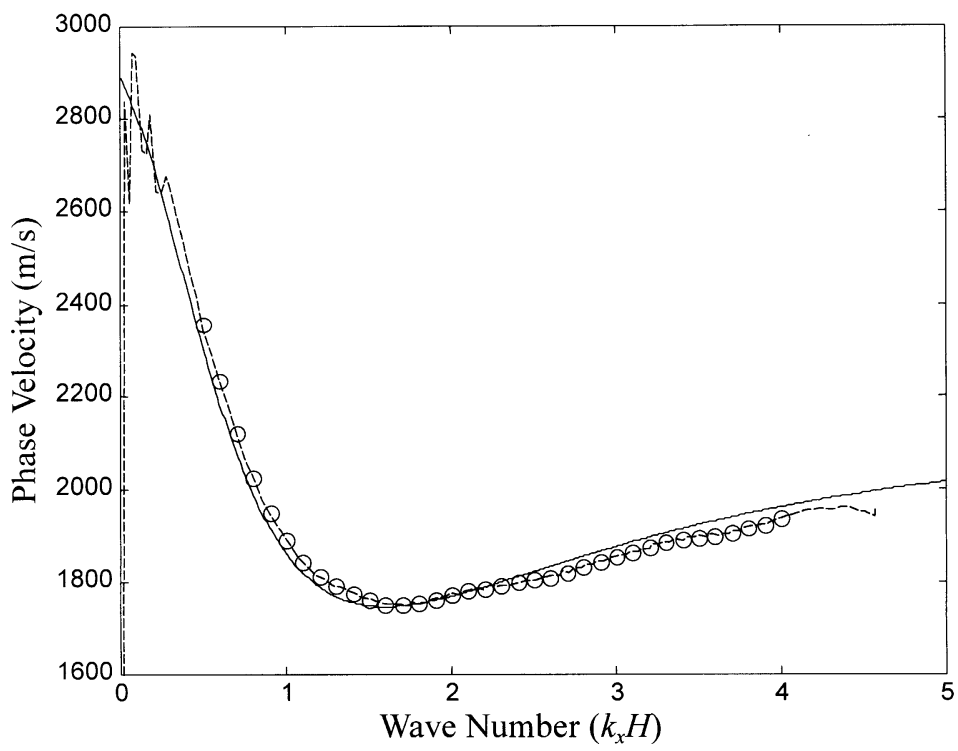

Fig. 10. Evaluation result of unbond ratio $\left(l_{1} / l=0 \%\right)$

$x_{2}=55 \mathrm{~mm}$, respectively. This shows that the distance between the two receivers are kept at constant spacing as $20 \mathrm{~mm}$, while the source to the receiver set is fixed at a distance equal to $35 \mathrm{~mm}$. A series of laser ultrasonic experiments was then conducted on the epoxy bonded copperaluminum layered specimen with $l_{1} / l$ equal to $0,40,65$ and $90 \%$. That is, the spacing of the receiver set is $0,40,65$ and $90 \%$ on the unbond area.

The dashed lines shown in Figs. 10-13 are the measured dispersion curves for unbond ratio $l_{1} / l$ equal to $0,40,65$ and $90 \%$, respectively. We note that for the case of $l_{1} / l$ equal to zero (Fig. 10), unlike the Lamb wave dispersion, the phase velocity of the generalized Rayleigh wave at the cut-off frequency is equal to that of an aluminum half-space. In this case, small noise on the phase angle measurement will cause large phase velocity error. And this explains why the phase velocities in Figs. 10 and 11 for the low wave number range are not accurate. The 36 features picked from experimental dispersion are indicated in open circles in Figs. 10-13.

\section{Evaluation results}

\subsection{Evaluation of bonding thickness}

To demonstrate the performance of the neural network method in predicting the bonding thickness, the neural

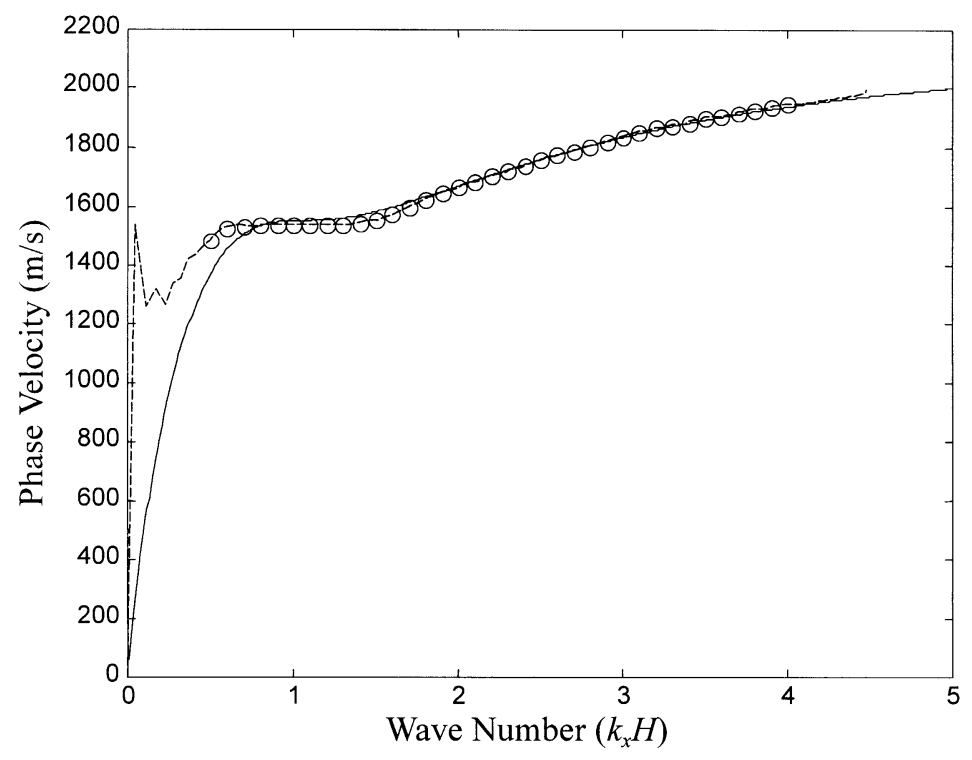

Fig. 11. Evaluation result of unbond ratio $\left(l_{1} / l=40 \%\right)$ 


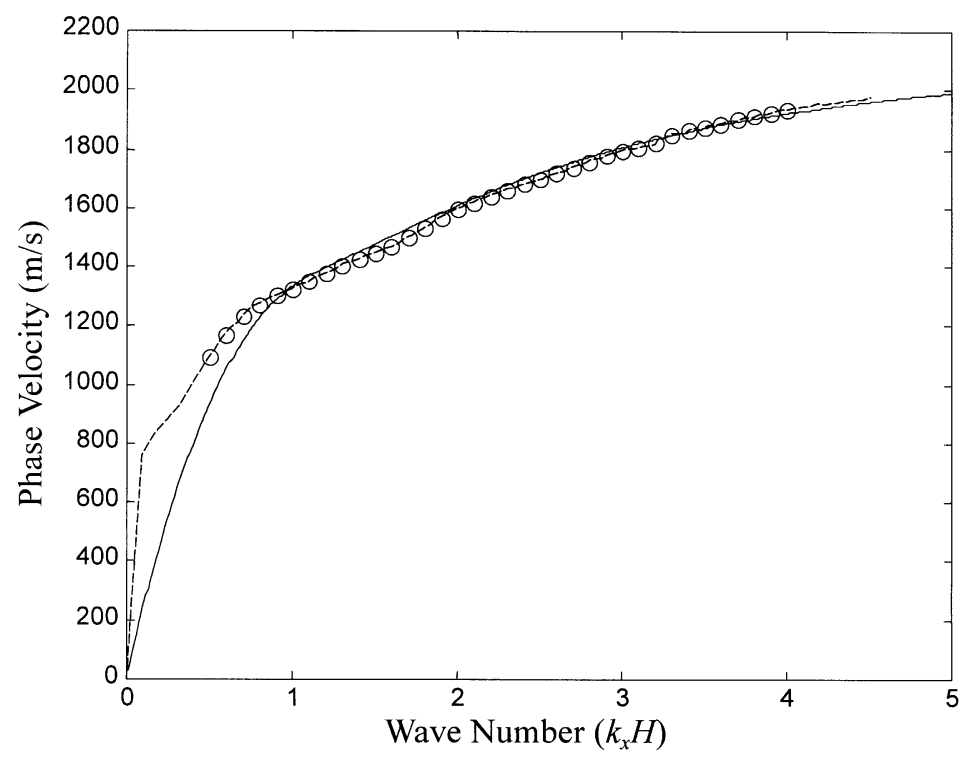

Fig. 12. Evaluation result of unbond ratio $\left(l_{1} / l=65 \%\right)$

network results are compared with those obtained by the simplex inverse method [15]. The error function indicating the difference between the measured $\left(v_{\mathrm{m}}\right)$ and the guessed $\left(v_{\mathrm{g}}\right)$ phase velocities is defined as

$e=\frac{\sum_{i=1}^{N}\left[v_{\mathrm{m}}(i)-v_{\mathrm{g}}(i)\right]^{2}}{\sum_{i=1}^{N}\left[v_{\mathrm{m}}(i)\right]^{2}}$

where $i$ represents the discrete non-dimensional wave number and $N$ is the number of data points utilized in the inversion process. In this paper, the 36 sampled velocities picked by neural network were also used here, i.e. $N=36$.
Shown in Table 1 are the comparison of the results obtained by the neural networks and the simplex inverse methods. The neural networks indicate that the epoxy thickness is of $0.099 \mathrm{~mm}$, while the simplex method indicates that the epoxy thickness is of $0.094 \mathrm{~mm}$. Although the determined values of the bonding thickness are close, the simplex inverse method requires 26 iterations or 52 forward calculations of the dispersion curves. On the other hand, the neural network method predicts the result in real time, and no forward calculation is required.

\subsection{Evaluation of unbond ratio}

Table 2 demonstrates the result of unbond ratio detection.

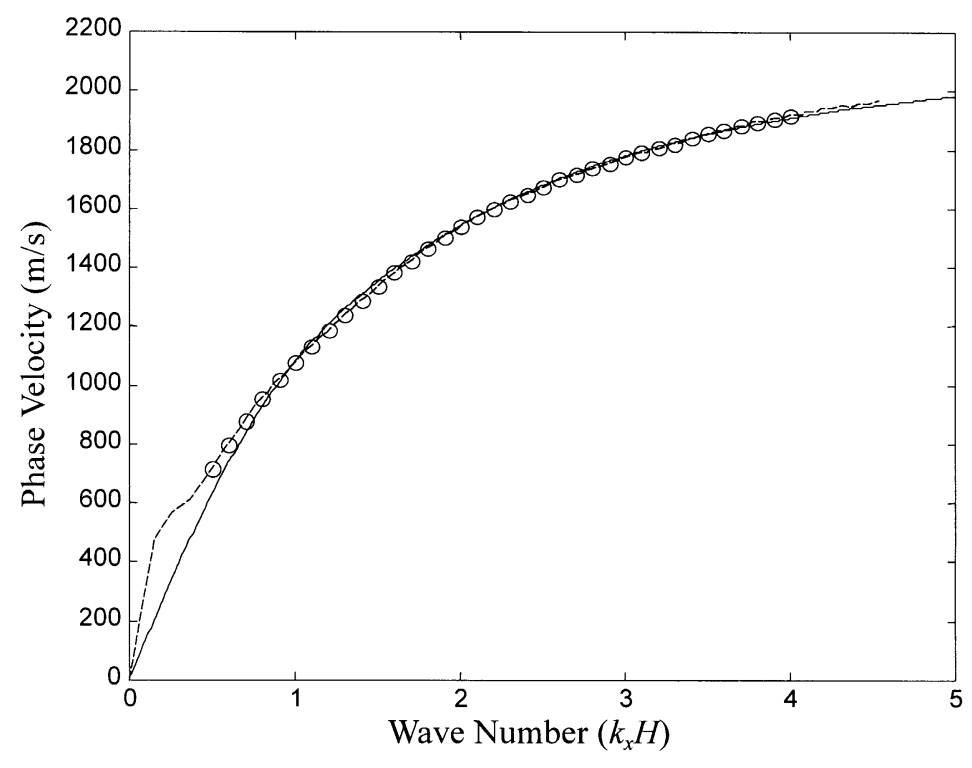

Fig. 13. Evaluation result of unbond ratio $\left(l_{1} / l=90 \%\right)$ 
Table 1

Results of bonding thickness evaluation

\begin{tabular}{lllllll}
\hline Epoxy thickness $(H)$ & \multicolumn{2}{l}{ Neural network } & & \multicolumn{2}{l}{ Simplex method } & \\
\cline { 2 - 3 } & Epoxy thickness $(H)$ & Error function $\left(\times 10^{-4}\right)$ & & Epoxy thickness $(H)$ & Error function $\left(\times 10^{-4}\right)$ & Iteration \\
\hline 0.1 & 0.0992 & 1.727 & & 0.0937 & 1.551 & 26 \\
\hline
\end{tabular}

Table 2

Results of unbond ratio evaluation

\begin{tabular}{lllllll}
\hline$l_{1} / l(\%)$ & \multicolumn{2}{l}{ Neural network } & & & \multicolumn{2}{l}{ Simplex method } \\
\cline { 2 - 3 } & $l_{1} / l(\%)$ & Error function $\left(\times 10^{-4}\right)$ & & $l_{1} / l(\%)$ & Error function $\left(\times 10^{-4}\right)$ & Iteration \\
\hline 0 & 0 & 1.778 & 0 & 1.778 & 22 \\
40 & 43.6 & 2.251 & 61.2 & 1.544 & 26 \\
65 & 67.5 & 6.042 & 63.7 & 4.057 & 28 \\
90 & 91.6 & 1.963 & 90.2 & 1.709 & 29 \\
\hline
\end{tabular}

Note that for a well bonded area, both the neural networks and the simplex method indicate the unbond ratio is zero. The results showed that accuracy of the unbond ratio predicted by the neural networks is slightly less than that of the simplex inverse method. However, the computer time needed in the neural networks is far less than that of the simplex inverse method. The solid lines in Figs. 10-13 are obtained by the forward calculation of dispersion curves with the unbond ratios determined by the neural networks. It is seen that the calculated dispersion curves are in good accordance with the measured ones (dashed lines).

\section{Conclusions}

In this paper, we have introduced the radial basis neural networks to evaluate the thickness of a bonding layer and/or unbond ratio of a bonded structure. Numerical simulations of surface wave dispersion in layered media were performed and the results were utilized to form the training and testing sets of the neural networks. Laser ultrasonic experiments were performed on an epoxy bonded copper-aluminum layered specimen with small bonding thickness. The output dispersive elastic wave signals were processed via the spectral analysis method to obtain the experimental dispersion curve. The results of this study have demonstrated that neural networks could be adopted for on-line evaluation of bonding thickness and unbond ratio of a bonded layered structure.

\section{Acknowledgements}

The authors thank the financial support of this research from the National Science Council of ROC through the grant NSC89-2212-E-002-009.

\section{References}

[1] Hutchins DA, Lundgren K, Palmer SB. A laser study of the transient Lamb waves in thin materials. J Acoust Soc Am 1989;85(4):1441-8.

[2] Dewhurst RJ, Edwards C, Mckie ADW, Palmer SB. Estimation of the thickness of thin metal sheet using laser generated utrasound. Appl Phys Lett 1987;51:1066-8.

[3] Nakano H, Nagai S. Laser generation of anti-symmetric Lamb waves in thin plates. Ultrasonics 1991;29:230-4.

[4] Veidt M, Sachse W. Ultrasonic point-source/point-receiver measurements in thin specimens. J Acoust Soc Am 1994;96(4):2318-26.

[5] Wu TT, Chai JF. Propagation of surface waves in anisotropic solids: theoretical calculation and experiment. Ultrasonics 1994;32(1):21-9.

[6] Wu T-T, Chen YC. Dispersion of laser generated surface waves in an epoxy-bonded layered medium. Ultrasonics 1996(34):793.

[7] Castagnede B, Kim KY, Sachse W, Thompson MO. Determination of the elastic constants of anisotropic materials using laser-generated ultrasonic signals. J Appl Phys 1991;70(1):150-7.

[8] Doyle PA, Scala CM. Ultrasonic measurement of elastic constants for composite overlays. Rev Prog QNDE 1991;10B:1453-9.

[9] Chai JF, Wu TT. Determinations of anisotropic elastic constants using laser generated surface waves. J Acoust Soc Am 1994;95(6):323241.

[10] Johnson MA, Berthelot YH, Brodeur PH, Jacobs LA. Investigation of laser generation of Lamb waves in copy paper. Ultrasonics 1996;34:703-10.

[11] Lorenz M, Willinga TS. Ultrasonic characterization of defects in steel using Multi-SAFT imaging and neural networks. NDT\&E Intern 1993;26(3):127-33.

[12] Kitahara M, Achenbach JD, Guo QC, Peterson ML, Ogi T, Notake M. Depth determination of surface-breaking cracks by a neural network. In: Thompson DO, Chimenti DE, editors. Review of Progress in Quantitative NDE, 10A. New York: Plenum Press, 1991. p. 689.

[13] Kitahara M, Achenbach JD, Guo QC, Peterson ML, Notake M, Takadoya M. Neural network for crack-depth determination from ultrasonic back scattering data. In: Thompson DO, Chimenti DE, editors. Review of Progress in Quantitative NDE, 11A. New York: Plenum Press, 1991. p. 701.

[14] Williams TP, Gucunski N. Neural networks for back calculation of moduli from SASW test. J Comp Civil Engng ASCE 1995(9(1):1):8.

[15] Wu TT, Liu YH. Inverse determinations of thickness and elastic properties of a bounding layer using laser-generated surface waves. Ultrasonics 1999;37:23-30.

[16] Wu TT, Chen YY. Analyses of laser generated surface waves in 
delaminated layered structures using wavelet transform. J Appl Mech ASME 1999;66(2):507-13.

[17] Aki K, Richards PG. Quantitative seismology: theory and methods. San Francisco: WH Freeman, 1980.

[18] Mal AK. Wave propagation in layered composite laminates under periodic surface loads. Wave Motion 1988;10:257-66.

[19] Braga MB. Wave propagation in anisotropic layered composites. PhD dissertation, Stanford University, 1990.
[20] Yuan D, Nazarian S. Automated surface wave method: inversion technique. J Geotech Engng 1993;119(7):1112-26.

[21] Liu P-L, Luo Y-F, Sun S-J. Intelligent bridge monitoring system. Proceedings of the 1996 ASCE Engineering Mechanics Conference, Fort Lauderdale, 1996.

[22] Chen S, Cowan CFN, Grant PM. Orthogonal least squares learning algorithm for radial basis function networks. IEEE Trans Neural Networks 1991;2:302-9. 